www.jmscr.igmpublication.org

Impact Factor 5.84

Index Copernicus Value: 83.27

ISSN (e)-2347-176x ISSN (p) 2455-0450

crossref DOI: _https://dx.doi.org/10.18535/jmscr/v5i2.123

Journal Of Medical Science And Clinical Research

IGM Publication

An Official Publication of IGM Publication

\title{
Early Brain Metastasis in a Case of Aggressive Renal Cell Carcinoma: A Case Report
}

Authors

Ramakant Tiwari, Rahat Hadi, Ashish Singhal, Satyajeet Rath

Dr. Ram Manohar Lohia Institute of Medical Sciences, Lucknow - 226010, UP

\begin{abstract}
Renal cell carcinoma (RCC) accounts for approximately $2 \%$ of malignancies worldwide with about 3,00,000 cases diagnosed per year and 1,00,000 deaths with the highest incidence occurring in Western countries. ${ }^{[1]}$ Brain metastasis from RCC occurs approximately in $8 \%$ of patients with metastatic RCC ( $m R C C)$. The guidelines from the National comprehensive cancer network (NCCN), European Association of Urology (EAU), and American Urological Association (AUA) recommend cerebral imaging only in patients who have symptomatology. The purpose of our case report was to review the presenting symptomatology and to assess prognostic factors for early brain metastasis followed by their overall treatment and survival. Patients presenting with brain metastases from renal cell carcinoma portend a poor prognosis, with a reported median survival of 4-6 months. Here, we present an interesting case report of 58years old male diagnosed as renal cell carcinoma, underwent Right Radical nephrectomy and developed brain metastases within a month of surgery.
\end{abstract}

Keywords: Brain metastasis, renal cell carcinoma, metastatic RCC.

\section{Introduction}

RCC is the most lethal of the urologic malignancies, with approximately $20-30 \%$ of patients with RCC presenting with metastatic disease and more than $40 \%$ of patients eventually dying from it. ${ }^{[2,3]}$ Approximately $4-17 \%$ of metastatic RCCs (mRCC) involve the brain, however, in $>50 \%$ of patients with cerebral metastases from RCC, multiple tumors are present. ${ }^{[3]}$ The majority of these patients present metastases at other sites, most commonly in the lung. ${ }^{[3]}$ Surgical resection, whole-brain radiotherapy (WBRT), or the combination of these modalities has been used as common treatment modalities. ${ }^{[4]}$

However, RCC is notorious for its resistance to conventional radiotherapy. ${ }^{[4]}$ Survival after
WBRT for brain metastases from RCC has not been very satisfactory: the median survival time after WBRT as a single treatment modality was shown to be a mere 4 months in a study. ${ }^{[5]}$ In addition, surgical resection is recommended only in the limited cases with a single brain metastasis, even if the metastasis is in an accessible location. ${ }^{[6]}$ Because of the lack of an effective treatment for this chemo- and radio-resistant malignancy, the prognosis of these patients is often poor. Brain involvement implies possible neurological sequelae and may decrease the quality of life of the patients. Identification of the cancer at an early stage is important because depending on what stage the patient is in, outcome, treatment, and survival rate can be determined. Early detection and surgical resection are the two key elements in 
a longer survival rate of a patient with metastatic RCC. Imaging is essential in early detection and helps to further diagnose RCC.

\section{Case Report}

The patient is a 58 year old male with no medical risk factors initially evaluated for complaint of recurrent fever, pain and swelling in right lumbar region. Ultrasonography of abdomen was done which showed mild hepatomegaly with largeheteroechoic mass lesion (108.6 x 69.2mm) seen in upper-middle pole of right kidney. Triphasic computed tomography scan abdomen was done which showed a large (approx.9.6 x 9.4 $\mathrm{x} 9.1 \mathrm{~cm}$ ) irregular heterogeneously enhancing lesion in mid part of right kidney. Lesion is bulging postero-medially and abutting the psoas muscle. Right renal vein \& IVC (Inferior vena cava) are patent. Right renal artery was partially encased by lymphadenopathy. Multiple enlarged lymphnodes seen at level of renal hilum and retrocaval region largest measuring approx. $2.7 \mathrm{x}$ $3.7 \mathrm{~cm}$.CECT Scan of Thorax was also done which showed multiple enlarged heterogeneously enhancing right pretracheal, bilateral hilarandsubcarinal lymph nodes with small ill defined lesions bilateral lower and right middle lobes. All the viral markers were negative. Patient also had anaemia for which multiple blood transfusion done (8 units). Patient underwent Right Radical nephrectomy. Post-operative histopathology reported renal cell carcinoma (Clear cell type), Fuhrman's nuclear grade-1, margins-clear, capsular break- not evident, hilar lymph node metastasis was seen in one out of thirteen, no pericapsular invasion or extranodal invasion, the final staging being pT2aN1.

Patient developed right sided hemiparesis after three weeks of surgery. A CECT Brain was performed after consulting neurology department, which showed multiple heterogeneously enhancing lesions in bilateral cerebral hemispheres (bilateral fronto-parietal region) with bony metastasis. Whole brain radiotherapy (WBRT) was planned with concurrent temozolamide, but only 7 fractions of radiation could be done.
Radiation had to be stopped due to poor general condition of the patient and was given symptommatic treatment for seizures \& unconsciousness. The patient developed anuria, sepsis and expired within three weeks of development of brain metastasis.

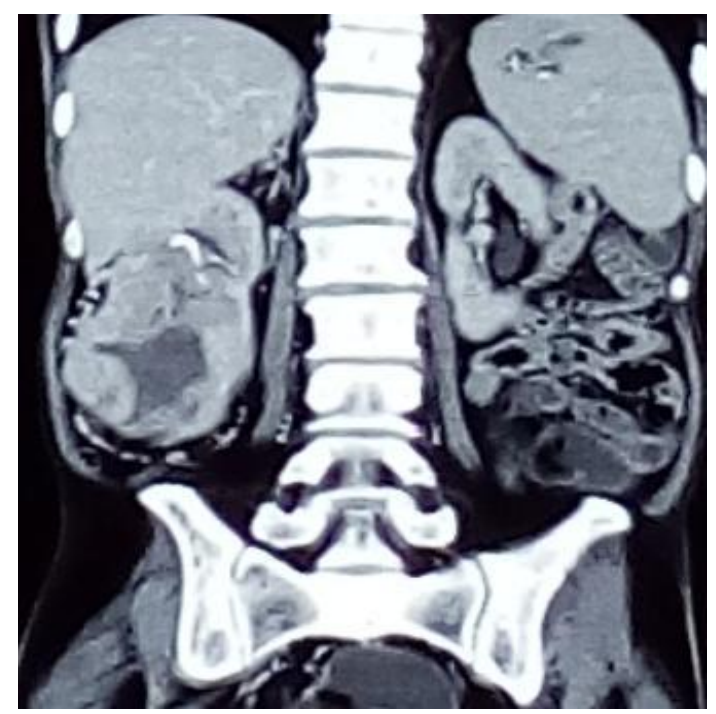

Image 1: Contrast enhanced CT Abdomen coronal view showing enlarged right renal mass

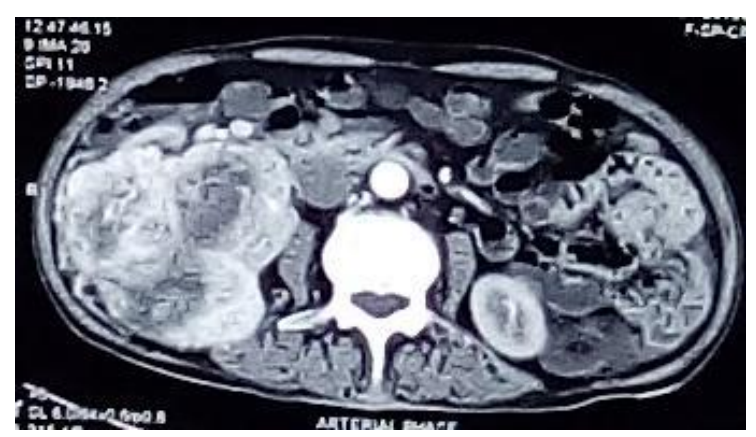

Image 2: Contrast enhanced CT Abdomen axial view showing enlarged right renal mass along with multiple enlarged lymph nodes

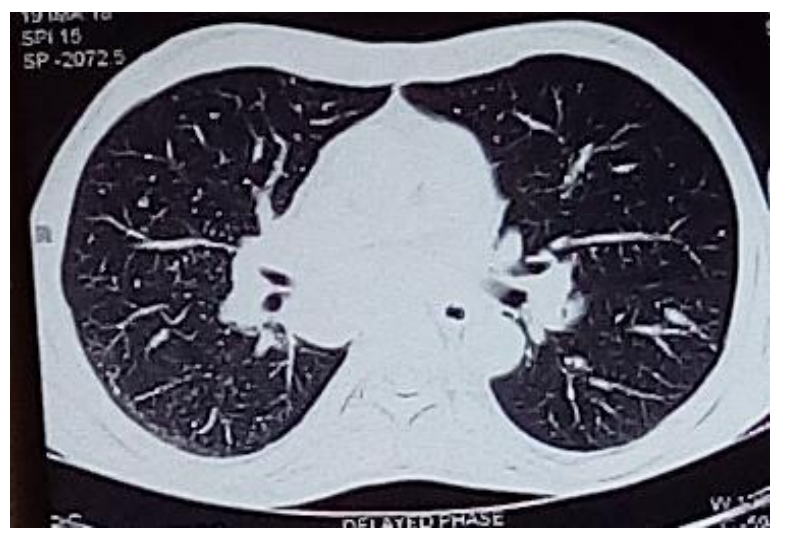

Image 3: Contrast enhanced CT Thorax axial view showing multiple enlarged lymph nodes 




Image 4: Contrast enhanced CT Brain showing multiple heterogeneously enhancing lesions in bilateral fronto-parietal region with surrounding edema

\section{Discussion}

RCC has been shown to metastasize to almost all soft tissues in the body, but most commonly to the lung, followed by bone, liver, brain, and local recurrence. ${ }^{[7]}$ Metastases to brain, bone, and liver often present as widely disseminated disease. Current guidelines for metastatic renalcell carcinoma (mRCC) do not recommend routine brain imaging as part of the surveillance protocol unless central nervous system (CNS) symptoms or abnormal laboratory values suggest brain involvement. Strict vigilance, high degree of clinical suspicion and prompt diagnostic tests are a must to diagnose and treat metastasis in early stages. Generally, in all RCC types, prognosis worsens with stage and Furham's histopathological grade. Of the three major histological subtypes of RCC that exist; conventional (clear cell) (80-90\%); papillary (10-15\%); and chromophobe (4-5\%);cRCC have a worse prognosis compared with papillary type (pRCC) and chromophobe type (chRCC) ${ }^{[8]}$

Studies have investigated patient- and tumorrelated prognostic factors for survival using multivariate analyses ${ }^{[9]}$. Performance status, interval from first cancer diagnosis to brain metastasis, extracranial metastasis, age, and single vs. multiple brain metastases were significant factors for survival. In the present case, evidence of a multiple brain metastasis, extracranial metastasis, and an interval less than one year from the diagnosis of RCC to brain metastases were associated with decreased survival.

The latest EAU guidelines for RCC have suggested the use of cytoreductive nephrectomy and metastasectomy in patients with mRCC ${ }^{[10]}$. Radiotherapy can be used for patients with nonresectable brain metastatic lesions; however, renal cell carcinoma is relatively radio-resistant. Unfortunately, the combination of these modalities has not been extensively discussed in the literature. According to the results of a retrospective study, surgical resection and/or stereotactic radiosurgery for brain metastases should be considered in single or limited lesions, because these improve the overall survival of patients compared with whole brain radiotherapy alone. ${ }^{[9]}$

\section{Conclusion}

$\mathrm{RCC}$, like any other cancer, is very unpredictable. There are many factors that must be identified in order to diagnose the type and stage of RCC, which, in turn, will assist in successfully treating the cancer. Although there are different treatment options available, this cancer is dependent on the patient's health, the stage of RCC, and continual follow up imaging. As shown in the above cases, prognosis and survival rate is reliant on how the patient's body responds to surgery and treatment. The current urologic guidelines may be missing a subset of metastatic RCC patients who could potentially benefit from early radiation or neurosurgical intervention. This may result in improved overall survival. Early detection will offer the longest survival rate; however, with a disciplined follow up regimen, continuous imaging, and surgical resection of tumors, the patient has a more promising chance of survival.

\section{References}

1. Ferlay J, Shin HR, Bray F, et al. Estimates of worldwide burden of cancer in 2008: GLOBOCAN 2008. Int J Cancer 2010; 127:2893-2917.

2. Jayson M, Sanders H. Increased incidence of serendipitously discovered renal cell carcinoma. Urology 1998 Feb;51(2):203-5. 
3. Jemal A, Murray T, Ward E, et al. Cancer statistics, 2005. CA Cancer J Clin. 2005;55:10-30.

4. T. Shuto, S. Inomori, H. Fujino, et al. Gamma knife surgery for metastatic brain tumors from renal cell carcinoma $\mathrm{J}$ Neurosurg, 105 (2006), pp. 555-560

5. M. Wronski, M.H. Maor, B.J. Davis, et al. External radiation of brain metastases from renal carcinoma: A retrospective study of 119 patients from the M. D. Anderson Cancer CenterInt J RadiatOncolBiolPhys, 37 (1997), pp. 753-759

6. Muacevic, M. Siebels, J.C. Tonn, et al. Treatment of brain metastases in renal cell carcinoma: Radiotherapy, radiosurgery, or surgery.

7. Lam JS, Leppert JT, Figlin RA, Belldegrun AS. Surveillance following radical or partial nephrectomy for renal cell carcinoma. CurrUrol Rep. 2005;6:7-18.

8. Capitanio U, Cloutier V, Zini L, et al. A critical assessment of the prognostic value of clear cell, papillary and chromophobe histological subtypes in renal cell carcinoma: a population-based study. BJU Int 2009 Jun;103(11)

9. Nieder C, Spanne O, Nordoy T, et al. Treatment of brain metastases from renal cell cancer. UrolOncol, 29 (2011), pp. 405-410.

10. Ljungberg, N.C. Cowan, D.C. Hanbury, M. Hora, M.A. Kuczyk, A.S. Merseburger, et al. EAU guidelines on renal cell carcinoma: the 2010 updateEurUrol, 58 (2010), pp. 398-406. 